\title{
Design for On-Sun Evaluation of Evaporator Receivers
}

\author{
Donald A. Jaworske ${ }^{1}$ \\ NASA Glenn Research Center, Cleveland, Ohio, 44135 \\ Anthony Colozza ${ }^{2}$ \\ Analex Corporation, Cleveland, Ohio, 44135 \\ and \\ Edward A. Sechkar ${ }^{3}$ \\ ASRC Aerospace Corporation, Cleveland, Ohio, 44135
}

\begin{abstract}
A heat pipe designed for operation as a solar power receiver should be optimized to accept the solar energy flux and transfer this heat into a reactor. Optical properties of the surface, thermal conductance of the receiver wall, contact resistance of the heat pipe wick, and other heat pipe wick properties ultimately define the maximum amount of power that can be extracted from the concentrated sunlight impinging on the evaporator surface. Modeling of solar power receivers utilizing optical and physical properties provides guidance to their design. On-sun testing is another important means of gathering information on performance. A test rig is being designed and built to conduct on-sun testing. The test rig is incorporating a composite strip mirror concentrator developed as part of a Small Business Innovative Research effort and delivered to NASA Glenn Research Center. In the strip concentrator numerous, lightweight composite parabolic strips of simple curvature were combined to form an array $1.5 \mathrm{~m} \times 1.5 \mathrm{~m}$ in size. The line focus of each strip is superimposed in a central area simulating a point of focus. A test stand is currently being developed to hold the parabolic strip concentrator, track the sun, and turn the beam downward towards the ground. The hardware is intended to be sufficiently versatile to accommodate on-sun testing of several receiver concepts, including those incorporating heat pipe evaporators. Characterization devices are also being developed to evaluate the effectiveness of the solar concentrator, including a receiver designed to conduct calorimetry. This paper describes the design and the characterization devices of the on-sun test rig, and the prospect of coupling the concentrated sunlight to a heat pipe solar power receiver developed as part of another Small Business Innovative Research effort.
\end{abstract}

\section{Nomenclature}

$C_{p} \quad=$ heat capacity

$d T / d t \quad=$ change in temperature over time

$I_{s} \quad=$ incident solar radiation

$M_{c} \quad=$ mass of copper

$P_{i} \quad=$ incoming power

$\mathrm{Vm} \quad=$ measured voltage

\section{Introduction}

TN-SITU resource utilization (ISRU) will require power to convert resources into products. Though the particular power requirements will depend on the ISRU chemistries and temperature regimes involved, ${ }^{1}$ one can envision

\footnotetext{
${ }^{1}$ Physicist, Space Environment and Experiments Branch, 21000 Brookpark Road/Mail Stop 309-2, AIAA Member.

${ }^{2}$ Research Engineer, Electrochemistry Branch, 21000 Brookpark Road/Mail Stop 309-1, non-member.

${ }^{3}$ Mechanical Engineer, Space Environment and Experiments Branch, 21000 Brookpark Road/Mail Stop 309-2, nonmember.
} 
two basic sources of power, solar or nuclear. Solar power is attractive for a number of ISRU applications because it is abundantly available, out to the orbit of Mars.

Applications may include reactions that produce oxygen from lunar regolith, where numerous different chemistry and temperatures regimes are required. Trash processing is another logistics area of interest where plastics are melted to create new re-tasked products such as radiation shielding bricks or reacted to create useful fuels. Again, numerous different chemistry and temperature regimes are under investigation and solar thermal processing may be beneficial.

Given that several potential ISRU applications utilize chemical reactions requiring heat, it is reasonable to explore the possibility of using concentrated sunlight as the source. Direct illumination of the external surface of a reactor or the internal surface of a receiver is inherently more efficient than converting solar energy to electricity with photovoltaics and then using the electricity to provide process heat.

Generic primary solar concentrators developed over the years include rigid and inflatable structures. Rigid concentrators offer excellent optics and high concentration ratios. Concentrator segments or facets are typically manufactured on a mandrel, coated with aluminum or silver using vapor deposition, and then mounted on a rigid support structure often made from box beams of aluminum or a polymer matrix composite. Depending on the mirror design, quality, and surface finish, rigid concentrators can achieve very high concentration ratios of approximately 8000 to $1 .^{2}$ However, to achieve this high concentration ratio the mirror shape needs to be very precise. Holding shape over wide temperature excursions is somewhat problematic, though careful selection of materials offers the prospect of near-zero thermal expansion structures. Manufacturing facets becomes increasingly difficult with size; and large single-element rigid concentrators are very expensive to manufacture.

Inflatable concentrators are often designed as a lens where one side is composed of a polymer film coated with a reflective coating, the other side is composed of a polymer film that is transparent, and the interior region is filled with a gas to form the shape of the lens. Other inflatable concentrators inflate an annulus which supports the reflective and clear polymer films used to reflect the incoming sunlight. For this type of concentrator to operate, maintaining an inventory of gas and a gas handling system is critical. Micrometeoroid impact is a constant threat mitigated through the use of make-up gas, which is in finite supply or by material that can harden into shape by exposure to solar UV radiation. ${ }^{3}$ Concentration ratios are considerably lower than rigid concentrators and the inflatable concentrator is often paired with a secondary concentrator to improve its performance. Inflatable concentrators combined with support structures and secondary concentrators are typically much less massive than rigid concentrator systems.

Secondary concentrators may be needed for either generic type of primary concentrator to increase the net concentration ratio and to redirect the energy to a more convenient location, including a fixed device decoupled from a sun-tracking concentrator device. Secondary concentrators developed over the years include compound reflecting and refractive types. ${ }^{2}$ In a compound secondary, concentration ratios can be improved by a factor of 4 or

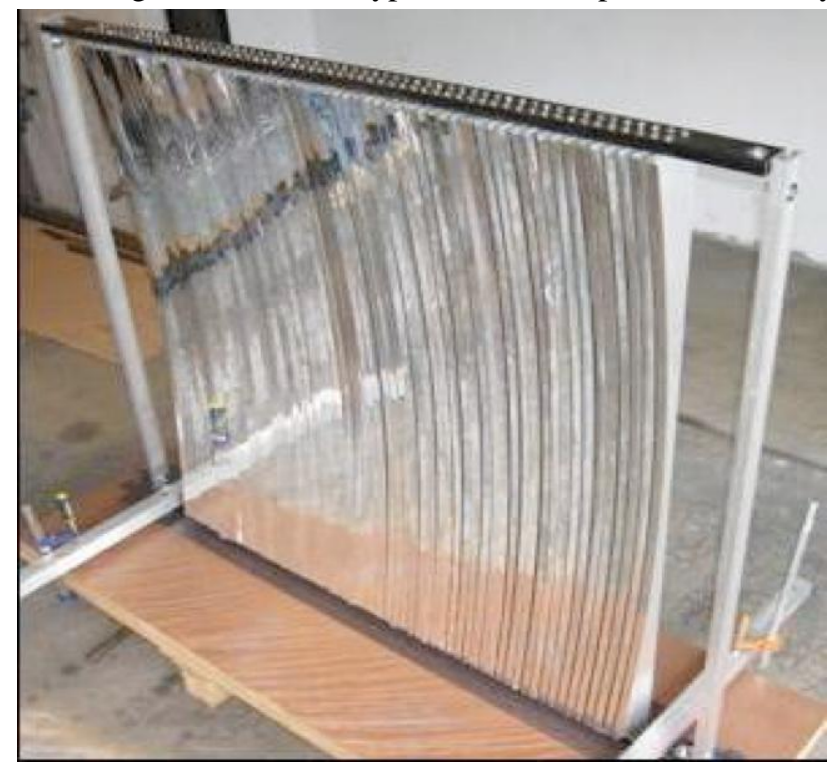

Figure 1. Strip mirror concentrator. more utilizing multiple reflections in a compound parabolic concentrator. However, less than perfect reflectivity results in heating of the secondary concentrator. Refractive secondary concentrators depend on total internal reflection to further concentrate the impinging sunlight. However, light absorbed by the imperfectly transparent media also results in substantial heating of the secondary concentrator risking the fracture of the refracting media.

DR Technologies, Inc. (now known as Vanguard Space Technologies), San Diego, CA, designed and built a strip concentrator for use in potential ISRU applications, as shown in Figure $1 .^{4}$ Built as part of a Small Business Innovative Research effort, the strip concentrator was delivered to NASA Glenn Research Center at the conclusion of the contract. The strip concentrator utilizes strips of polymer matrix composite bonded to curved pieces of a honeycomb structure. A reflective strip is bonded to the surface of the composite material. The strips are flat across the small dimension and curved to an approximately parabolic shape in the long dimension. The one-dimensional curvature of each 
strip provides some advantages. Light from the strip does not focus to a spot, it focuses to a rectangular region the width of the strip and much shorter than the strip length. Properly aligned, the line focus of each strip is superimposed in a central region of focus from which the concentrated sunlight can be captured and utilized. Manufacturing the essentially identical strips is simple leading to an economical concentrator system.

Utilizing concentrated solar power over significant operational times requires integrating the concentrator to a sun-tracking mechanism. A secondary optics system using lenses to collimate the sunlight and a mirror to turn the beam towards a receiver located in a position not shadowing the concentrator, could deliver the concentrated sunlight to an ISRU reactor or a heat pipe receiver.

Advanced Cooling Technologies, Inc., Lancaster, PA, designed and built dual pressure-controlled heat pipes (PCHPs) to allow heat collected in a centrally located receiver to be distributed to two ISRU furnaces. ${ }^{5}$ Built as part of a second Small Business Innovative Research effort, the PCHPs were delivered to NASA Glenn Research Center at the conclusion of the contract. Coupling the strip concentrator to the PCHP hardware may provide one avenue for conducting research on the integration of a solar concentrator with heat receiver hardware.

The purpose of this paper is to describe the integration of the strip mirror with a sun-tracking structure, a lens and mirror combination for collimating and turning the sunlight towards a receiver, and the quantification of the integrated system via characterization devices that are being developed to evaluate the effectiveness of the solar concentrator by calorimetry. The paper also considers the prospect of coupling the concentrated sunlight to a heat pipe solar power receiver.

\section{Design}

The design considerations focused on three basic requirements: tracking, mobility, and minimal shadowing of the concentrator, that is, minimizing any shadows created on the primary concentrator surface by secondary optics or heat receiver systems that reduce the solar flux passing through the overall system. Hence, the starting point for the design was the strip concentrator and the first requirement tackled was tracking the sun. Commercially available sun tracking equipment was reviewed and a small lightweight sun tracker was selected from Habi-Tek, Geneva, IL. The tracker offers two-axis operation, under both motorized or manual control. A photocell housed in a high aspect ratio (long/narrow) cylinder will serve as a means to adjust the tracking hardware initially and to confirm optimum sun tracking over the course of operation. The next requirement to be addressed was the need for mobility of the test bed. Envisioned to be portable, the concentrator will be moved outside for testing during daytime operations. A small trailer was selected to meet the mobility requirement. Jack stands at the four corners of the trailer will serve to stabilize the hardware upon deployment. The third requirement was the need to direct the beam downward toward the ground in order to minimize shadowing of the concentrator and to accommodate testing utilizing a calorimeter, other test coupons, or a heat pipe solar power receiver such as the PCHP furnace assembly. This requirement was met by utilizing a lens and mirror combination supported by a boom connected to the sun tracking hardware. The lens-mirror-lens combination serves to collimate the sunlight, direct the solar energy downward toward the ground, and direct the sunlight into a calorimeter, onto a test coupon, or into a receiver cavity.

Figure 2 summarizes the salient features of the concept. Sunlight impinging on the strip concentrator is directed

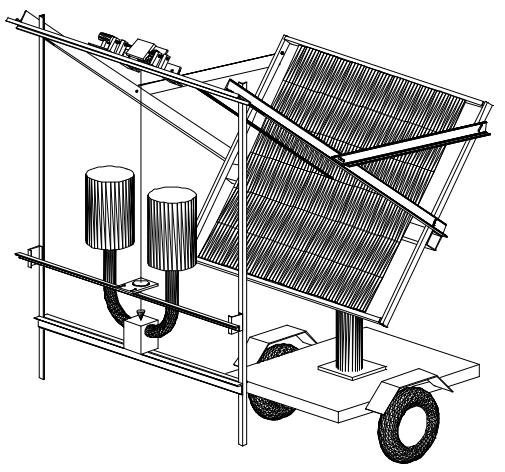

Figure 2. Strip concentrator, sun tracker and notional dual PCHP heat receiver on trailer. to a mirror and lens assembly located near the focus and supported by a boom. Hanging from the boom under the mirror is a second lens and space for a calorimeter, test article, or as shown, a notional heat pipe receiver. Not shown in Figure 2 is supporting hardware such as the photodiode for sun tracking verification or jack stands for stabilizing the platform when deployed. It should be noted that one of the key simplifying features of the design is that the receiver is carried with the movment of the sun tracker through the movement of the boom, accomplished by having the receiver hanging from the boom tip directly under the turning mirror.

The design of the lens and mirror combination to be located on the boom is shown notionally in Figure 3. The lens must be large enough to capture and collimate the concentrated sunlight and the mirror large enough to direct it downward. Because the lens and mirror are affixed to the boom, the mirror requires only one axis control to direct the collimated beam downward, achieved through the use of a stepper motor shown coaxial to the mirror pivot. 
Minimizing shadowing through the use of a lens and turning mirror assembly will help maximize the energy that is transmitted to the surface below. The overall losses imposed by the secondary lens and mirror system, through absorption and off-axis reflection, are estimated to be $10 \%$ of the total light reflected from the primary mirror.

We have developed characterization devices and instrumentation to evaluate the effectiveness of solar concentrators. The process for evaluating the solar concentrator involves ensuring that the system is pointing accurately at the sun, measuring the instantaneous solar flux available at the test location, and then measuring the power that passes through the concentrator system.

To determine the total efficiency of the concentrator system, a calorimeter is utilized to determine the total solar power being supplied by the concentrator and comparing that to the total solar power incident on the concentrator. The calorimeter is constructed from a

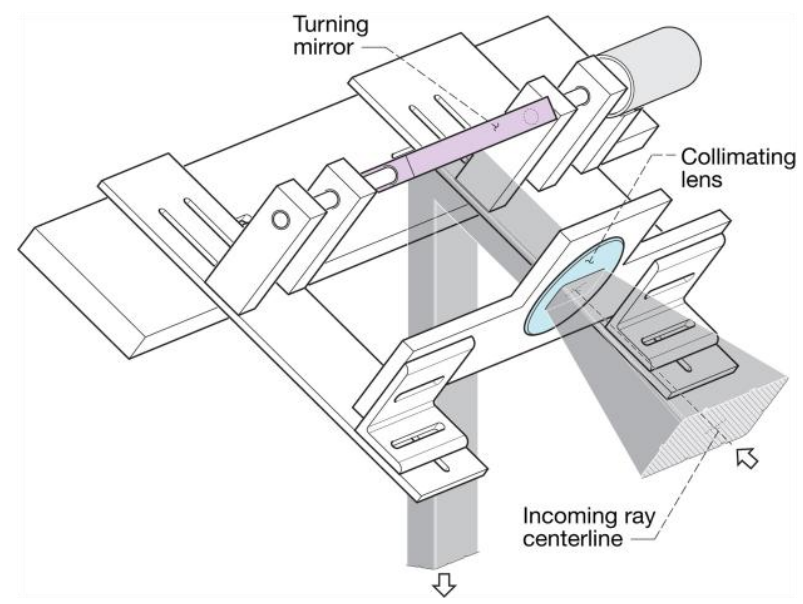

Figure 3. Notional lens and turning mirror assembly. copper chamber into which the concentrated sunlight is focused.

The cylinder is painted black using high temperature flat paint to reduce the reflection of light from within the

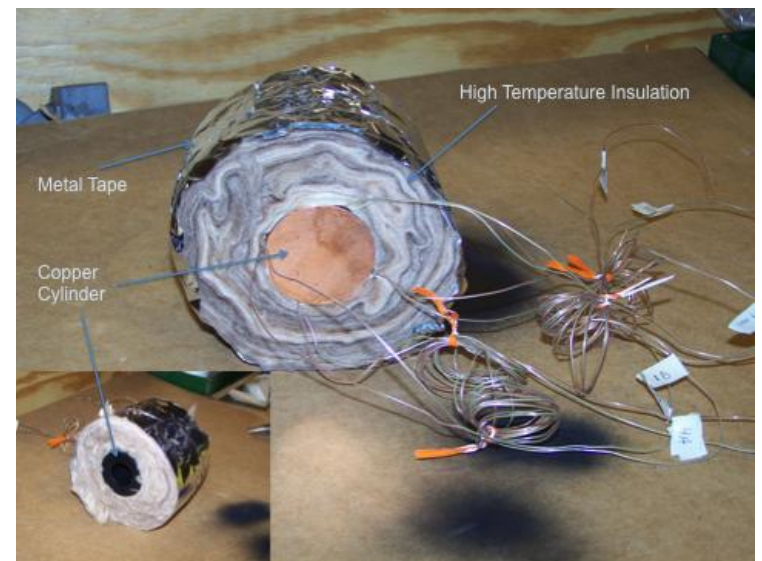

Figure 4. Cylinder side-wall insulation and thermocouple routing. chamber. Thermocouples are installed on the outside of the cylinder wall to measure the rise in temperature over time. The cylinder is wrapped in high temperature insulation and the thermocouple wires are routed through the bottominsulated end of the calorimeter, as shown in Figure 4. Insulation is then applied to the top and bottom of the cylinder and a hole was made on the topside, as shown in Figure 5, to allow the concentrated sunlight to enter the copper cylinder.

The calorimeter is placed at the focus of the concentrator so that the light begins to disperse as it enters the hollowed center. This is done to evenly disperse the light along the interior wall of the calorimeter as well as to prevent the highly concentrated sunlight from melting the copper.

Using the rise in temperature of the calorimeter over a short period of time, the incoming power $\left(P_{i}\right)$ to the calorimeter can be calculated, as given in Eq. (1). The slope of the temperature curve $(d T / d t)$ along with the specific heat capacity $\left(c_{p}\right)$ of pure copper, $384.9 \mathrm{~J} / \mathrm{kg}^{\circ} \mathrm{C}$, and mass $\left(M_{c}\right)$ are used to estimate the total power being transmitted by the concentrator system.

$$
P_{i}=M_{c} c_{p} \frac{d T}{d t}
$$

A calibrated photovoltaic cell, shown in Figure 6, is used to determine the incoming solar power incident on the concentrator. The calibrated photovoltaic cell is connected to a precision $1 \mathrm{ohm}$ resistor across which the voltage is measured. Since the resistor is $1 \mathrm{ohm}$ there is a 1 to 1 correlation between the measured voltage and current produced by the solar cell. The solar cell current output is linear with the incoming solar radiation. From this the incident solar radiation $\left(I_{\mathrm{s}}\right)$ can be determined from the measured voltage $\left(\mathrm{V}_{\mathrm{m}}\right)$, as given by Eq. (2).

$$
I_{s}=3.6 V_{m}
$$

The output of the solar cell can also be used to help fine tune the position of the concentrator while pointed toward the sun prior to initializing sun tracking. The calibrated cell is housed at the bottom of a tube which is

4

American Institute of Aeronautics and Astronautics 
mounted perpendicular to the plane of the concentrator and therefore points in the direction of the centerline of the concentrator. Two evaluations can be made utilizing the solar cell and calorimeter combination depending on the location of the calorimeter. First, by comparing the incident solar power from the photovoltaic cell to the transmitted power determined by the calorimeter located in front of the lens and turning mirror assembly, the overall efficiency of the primary concentrator can be determined. This measurement provides an indication of the quality of the mirrored surface. Second, it should be possible to place the calorimeter at the output of the lens-mirror-lens optical path near the location of the PCHP heat receiver. Placing the calorimeter at this location and comparing the incident solar power to the transmitted power should yield the overall efficiency of the combined primary and secondary optics system.

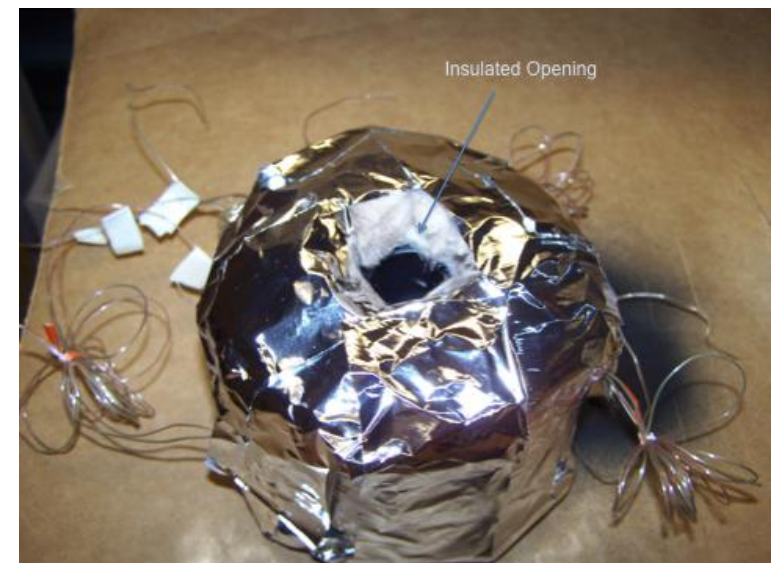

Figure 5. Fully insulated calorimeter with light opening.

\section{Conclusion}

In-situ resource utilization will require power to convert resources into products. A test rig is being designed and built to conduct on-sun testing, incorporating a composite strip concentrator that was recently developed as part of a Small Business Innovative Research effort and delivered to NASA Glenn Research Center. The test rig design considerations focused on three basic requirements: tracking the sun (a Habi-Tek solar tracker), mobility (a small

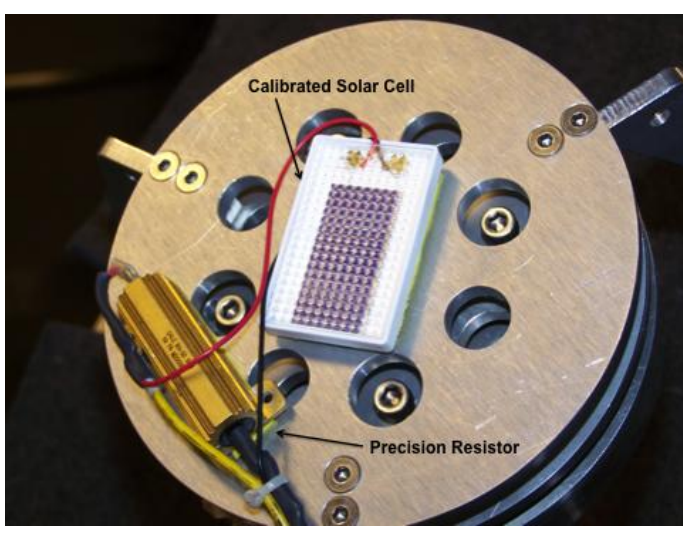

Figure 6. Installed calibrated solar cell. trailer), and minimal shadowing (a lens-mirror-lens system to relocate the heat receiver outside of the concentrator's focal region). Characterization devices are also needed to evaluate the effectiveness of the solar concentrator, and include a photovoltaic cell used to determine the solar power incident on the concentrator and a solid copper receiver designed to conduct calorimetry. The calorimetry will enable evaluation of the overall efficiency of the optical system for use in a variety of ISRU applications.

The concentrator system will also act as a test-bed to provide concentrated solar energy at a known power density to various applications. These applications will include material samples, process reactions, and technology demonstrations. In addition the system is versatile and can be used to evaluate different types of concentrator technologies and focusing designs. These items can include: secondary concentrators such as different lenses and focusing schemes, optical transmission components such as fiber optics or refractive secondary concentrators, and light absorbing receiver cavities.

\section{Acknowledgments}

The authors acknowledge the Enabling Technology Development and Demonstration Program and the In-Situ Resource Utilization Project for their support of the ongoing work described here.

\section{References}

${ }^{1}$ Hepp, A.F., Linne, D.L., Landis, G.A., Wadel, M.F., and Colvin, J.E., "Production and Use of Metals and Oxygen for Lunar Propulsion," J. Propul. Power, Vol. 10, No. 6, 1994, pp. 834-840.

${ }^{2}$ Gordon, P.E., Colozza, A.J., Hepp, A.F., Heller, R.S., Gustafson, R., Stern, T. and Takashi, N., "Thermal Energy for Lunar In-situ Resource Utilization: Technical Challenges and Technology Opportunities," NASA TM-2011-217114, 2011.

${ }^{3}$ Freeland, R.E., Helms, R.G., Willis, P.B., Mikulas, M.M., Stuckey, W., Steckel, G. and Watson,J., "Inflatable Space Structures Technology Development for Large Radar Antennas", $55^{\text {th }}$ International Astronautical Congress, Vancouver, BC, Canada, IAC-04-IAF-1.1.10, 2004.

${ }^{4}$ Wyatt, S. and Stern, T., "Fresnel Reflector Development for High Temperature Solar-Fired Furnace in Space," $7^{\text {th }}$ International Energy Conversion Engineering Conference, Denver, CO, AIAA 2009-4559, 2009.

$$
5
$$

American Institute of Aeronautics and Astronautics 
${ }^{5}$ Hartenstine, J.R., Walker, K.L., Tarau, C., and Anderson, W.G., "Pressure Controlled Heat Pipe Solar Receiver for Regolith Oxygen Production with Multiple Reactors," 49th AIAA Aerospace Sciences Meeting, Orlando, FL, AIAA 2011-611, 2011. 